\title{
Comunicação
}

[Communication]

\section{Características eletrocardiográficas de éguas da raça Crioula em diferentes fases do período gestacional}

\author{
[Electrocardiographic features of Crioulo mares in different pregnancy stages] \\ G. Casa, M.D. Oliveira, C.R. Regianini, M. Lovatel, C.D.L. Cancelier, \\ M.G.N. Xavier, J. Volpato, M.E. Saito, L.A. Yonezawa*
}

Centro de Ciências Agroveterinárias (CAV), Universidade do Estado de Santa Catarina (UDESC) - Lages, SC

O cavalo Crioulo teve sua origem na Península Ibérica e, por meio dos colonizadores, a raça foi inserida na América do Sul, com destaque na região Sul do Brasil. Em quatro séculos de evolução, a seleção natural conferiu a esses animais características que envolvem rusticidade e resistência (História, 2017). A versatilidade da raça possibilitou a utilização desses animais não só para a pecuária, mas também para o ecoturismo, o esporte e a prestação de serviços.

A égua Crioula tem um período de gestação longo, variando de 312 a 364 dias, com média de $335,6 \pm 10,5$ dias (Winter et al., 2007). No período gestacional, ocorrem alterações fisiológicas adaptativas que visam fornecer condições adequadas para a manutenção e o desenvolvimento do feto (Barcellos, 2005). Desse modo, é muito importante acompanhar clinicamente os animais gestantes e assegurar sua saúde. Vários métodos podem ser empregados para o acompanhamento da gestação de éguas, entre eles o exame eletrocardiográfico.

O eletrocardiograma (ECG) constitui a ferramenta diagnóstica mais indicada para avaliar os fenômenos elétricos no coração (Yonezawa et al., 2014). Trata-se de um exame simples, de baixo custo, que pode ser realizado a campo, não invasivo e que não causa danos ao feto (Barcellos, 2005; Yonezawa et al., 2014). Por meio do exame eletrocardiográfico, é possível avaliar frequência, ritmo e tempo de condução do estímulo elétrico em equinos
(Yonezawa et al., 2014). Contudo, a influência da gestação sobre a atividade elétrica cardíaca ainda é pouco conhecida na espécie equina, e não há estudos sobre a raça Crioula. Dessa forma, o presente estudo objetivou avaliar o eletrocardiograma de éguas da raça Crioula em diferentes fases do período gestacional.

O estudo foi avaliado e aprovado pela Comissão de Ética no Uso de Animais (CEUA) da Universidade do Estado de Santa Catarina (UDESC), sob protocolo número 5341030717. Para tal, foram utilizadas éguas da raça Crioula, adultas, provenientes de uma mesma propriedade rural, situada no munícipio de Painel/SC, e submetidas ao mesmo manejo nutricional e sanitário. Os animais foram previamente submetidos à avaliação clínica, por meio de exame físico completo, hemograma e bioquímica sérica. Após a constatação da higidez, as éguas foram divididas em quatro grupos, conforme o período gestacional aproximado em que se encontravam: éguas não gestantes $(\mathrm{G} 0, \mathrm{n}=9)$; aos três meses de gestação $(\mathrm{G} 3, \mathrm{n}=11)$; aos seis meses de gestação (G6, n= 11); e aos 10 meses de gestação $(\mathrm{G} 10, \mathrm{n}=11)$, caracterizando-se os terços inicial, médio e final da gestação, respectivamente.

Para a realização do exame eletrocardiográfico, utilizou-se um eletrocardiógrafo computadorizado (ECG-PC Vet, TEB, Brasil). Os eletrodos tipo "jacaré" foram fixados na pele do animal com algodão umedecido com álcool $70 \%$ e posicionados conforme a técnica de base-

Recebido em 20 de dezembro de 2017

Aceito em 17 de abril de 2018

*Autor para correspondência (corresponding author)

E-mail: leticiay@gmail.com 
ápice (Patteson, 2002), tomando-se o cuidado de manter o animal com os membros paralelos entre si e perpendiculares ao eixo longitudinal do corpo. Os traçados foram obtidos na derivação bipolar D1, em sensibilidade de $\mathrm{N}(10 \mathrm{~mm} / \mathrm{mV})$ e na velocidade de $25 \mathrm{~mm} / \mathrm{s}$. Em seguida, foram realizadas a análise e a interpretação dos traçados eletrocardiográficos, avaliando-se a frequência cardíaca (FC), o ritmo e as mensurações de ondas e intervalos.

De acordo com a distribuição normal dos dados, analisada pelo teste de Kolgomorov e Smirnov, as variáveis foram avaliadas segundo métodos estatísticos paramétricos. Os dados foram inicialmente avaliados por meio de teste de análise de variância (ANOVA), e, quando significativos, as médias foram confrontadas pelo teste de múltipla amplitude de Tukey. As análises foram processadas com o auxílio do programa estatístico computadorizado (SigmaStat, versão 3.10), sendo consideradas significativas quando $\mathrm{P}<0,05$. As variáveis qualitativas de ritmo e conformação das ondas $\mathrm{P}$ foram comparadas entre os grupos de forma descritiva, conforme a frequência de ocorrência. Os valores dos parâmetros eletrocardiográficos dos grupos avaliados estão apresentados na Tab. 1.

Tabela 1. Média \pm desvio-padrão dos parâmetros eletrocardiográficos de éguas da raça Crioula não gestantes $(\mathrm{G} 0, \mathrm{n}=9)$ e durante o terceiro $(\mathrm{G} 3, \mathrm{n}=11)$, o sexto $(\mathrm{G} 6, \mathrm{n}=11)$ e o $10^{\circ}$ mês de gestação $(\mathrm{G} 10$, $\mathrm{n}=11$ )

\begin{tabular}{ccccc}
\hline Parâmetro & G0 & G3 & G6 & G10 \\
\hline FC (bpm) & $45,67 \pm 10,75^{\mathrm{a}}$ & $44,91 \pm 7,91^{\mathrm{a}}$ & $46,91 \pm 9,12^{\mathrm{a}}$ & $60,45 \pm 12,16^{\mathrm{b}}$ \\
Onda P (ms) & $122,33 \pm 30,61$ & $144,55 \pm 21,37$ & $140,82 \pm 20,68$ & $124,27 \pm 21,33$ \\
Onda P1(mV) & $0,28 \pm 0,06^{\mathrm{a}}$ & $0,18 \pm 0,06^{\mathrm{b}}$ & $0,17 \pm 0,05^{\mathrm{b}}$ & $0,19 \pm 0,08^{\mathrm{b}}$ \\
Onda P2 (mV) & $0,35 \pm 0,05$ & $0,31 \pm 0,08$ & $0,28 \pm 0,07$ & $0,32 \pm 0,08$ \\
Intervalo PR (ms) & $296,33 \pm 52,42$ & $295,18 \pm 44,26$ & $291,09 \pm 42,69$ & $272,91 \pm 53,96$ \\
QRS (ms) & $115,00 \pm 9,21^{\mathrm{a}}$ & $113,73 \pm 17,52^{\mathrm{a}}$ & $96,27 \pm 11,88^{\mathrm{b}}$ & $100,64 \pm 13,19^{\mathrm{ab}}$ \\
QRS (mV) & $-1,51 \pm 0,46^{\mathrm{a}}$ & $-1,09 \pm 0,18^{\mathrm{b}}$ & $-0,73 \pm 0,32^{\mathrm{c}}$ & $-0,84 \pm 0,22^{\mathrm{bc}}$ \\
Intervalo QT (ms) & $469,67 \pm 48,53^{\mathrm{ab}}$ & $490,36 \pm 53,64^{\mathrm{a}}$ & $451,82 \pm 51,34^{\mathrm{ab}}$ & $420,27 \pm 47,96^{\mathrm{b}}$ \\
\hline
\end{tabular}

FC: frequência cardíaca; bpm: batimento por minuto; ms: milissegundo; $\mathrm{mV}$ : milivolt.

Letras minúsculas diferentes na mesma linha indicam diferença estatística (teste de Tukey, P<0,05).

Os valores de frequência cardíaca encontrados nos grupos das éguas vazias (G0), aos três meses (G3) e aos seis meses de gestação (G6) se apresentaram dentro do intervalo para cavalos adultos em repouso descrito por Fregin (1992), cujos valores citados são de 26 a 50bpm, e próximos à média encontrada por Schade et al. (2014) para cavalos Crioulos $(43,6 \pm 11,6)$. Todavia, o valor obtido de G10 $(60,45 \pm 12,16 \mathrm{bpm})$ foi significativamente maior $(\mathrm{P}=0,002)$ em relação aos outros momentos, conforme demonstrado na Tab. 1. Fernandes et al. (2004) verificaram uma elevada FC média de $57,7 \pm 18,6 \mathrm{bpm}$ em 11 éguas gestantes da raça Puro Sangue Inglês, porém os autores não informaram em qual fase da gestação os animais estavam. Schade et al. (2014) também verificaram maior valor de FC em 27 éguas Crioulas no terço médio de gestação $(52,9 \pm 10,4 \mathrm{bpm})$, quando comparadas a 43 éguas não gestantes $(43,7 \pm 9,9 \mathrm{bpm})$. Em contrapartida, Pascon et al. (2015) verificaram resultados semelhantes em 34 éguas Crioulas gestantes
$(54,84 \pm 8,39)$ e 50 não gestantes $(52,11 \pm 8,73)$, no entanto também não informaram em qual período gestacional. De acordo com Picon e Sá (2005), mulheres gestantes apresentam alterações hemodinâmicas que incluem aumento da volemia e do débito cardíaco, além de diminuição da resistência vascular sistêmica. A hipervolemia surge para suprir a demanda do útero, bem como para proteger a mãe da perda sanguínea durante o parto. Adicionalmente, o débito cardíaco se eleva devido ao aumento do volume sistólico e da FC, o que implica maior perfusão em diversos órgãos e tecidos, como útero e placenta, garantindo aporte sanguíneo para o feto em crescimento, além de rins, pele, glândula mamária, intestinos e outros (Picon e Sá, 2005). Como a maior taxa de crescimento fetal ocorre no terço final da gestação da égua (Sellnow, 1999), no presente estudo, acredita-se que a elevação da FC ocorreu nessa fase para aumentar o débito e garantir suprimento sanguíneo para o incremento do desenvolvimento fetal. 
Quanto ao ritmo cardíaco, na maioria das éguas foi verificado o ritmo sinusal normal. Segundo Patteson (2002), considera-se taquicardia sinusal quando o ritmo for sinusal de origem e a frequência cardíaca ultrapassar $60 \mathrm{bpm}$. A taquicardia sinusal foi encontrada em 1/9 (11\%) animais do G0, 1/11 (9\%) do G3, 1/11 (9\%) do G6 e 6/11 (55\%) do G10, semelhantemente ao verificado por Fernandes et al. (2004), em 30\% das éguas gestantes avaliadas da raça Puro Sangue Inglês. A taquicardia sinusal verificada nas éguas do grupo G10 pode ser justificada pelo aumento da FC decorrente do acréscimo na demanda circulatória no terço final da gestação, conforme discutido anteriormente. Para os animais dos demais grupos (G0, G3 e G6), esse ritmo pode ser justificado pela excitação dos animais no momento do exame e pelo consequente aumento da FC (Patteson, 2002), uma vez que as éguas eram mantidas em sistema extensivo com pouco manejo.

De acordo com Diniz et al. (2011), quando comparados às demais espécies de animais domésticos, os equinos saudáveis podem apresentar alta incidência de arritmias cardíacas e distúrbios de condução, sendo muitos considerados fisiológicos quando atribuídos ao tônus vagal elevado, como bloqueios atrioventriculares de primeiro e segundo graus, arritmia sinusal e bradicardia sinusal (Fernandes et al., 2004; Yonezawa et al., 2014). Contudo, no presente estudo, não foram detectados maiores distúrbios de ritmo ou de condução.

A maioria dos animais apresentou a onda $\mathrm{P}$ com morfologia bífida, o que é um achado normal na espécie equina (Schade et al., 2014; Yonezawa et al., 2014). Não houve diferença entre os grupos quanto à duração da onda $\mathrm{P}$, como também não houve diferença para o valor do intervalo PR. Foi verificado um maior valor $(\mathrm{P}=0,003)$ da amplitude de P1 do grupo de éguas vazias comparado aos grupos de éguas gestantes (G3, G6 e G10), entretanto, como o valor médio de P1 se aproxima do valor de $\mathrm{P} 2$ em todos os grupos, de modo geral, acredita-se que essa diferença estatística encontrada não seja clinicamente relevante.

Com relação à duração do complexo QRS, verificou-se menor valor do G6 quando comparado aos grupos G0 e G3 (P=0,005). A duração do complexo QRS está relacionada ao tempo necessário para despolarização ventricular. Embora não se saiba a causa para o G6 apresentar esse menor valor, sabe-se que a avaliação do complexo QRS para determinar o tamanho cardíaco é limitada (Fernandes et al., 2004). Isso se deve ao fato de as fibras de Purkinje se apresentarem mais profundamente no miocárdio, de modo que a maior parte do miocárdio ventricular se despolariza simultaneamente, sem a ativação seriada das células, não sendo possível estabelecer uma correlação do complexo QRS com as dimensões das câmaras ventriculares (Yonezawa et al., 2014). Quanto à amplitude do complexo QRS, verificou-se diferença significativa entre os grupos $(\mathrm{P}<0,001)$, tendo o G0 apresentado o maior valor, o G3 valor intermediário, e G6 e G10 os menores valores. Schade et al. (2014) também verificaram menor amplitude de QRS de éguas Crioulas gestantes comparadas às não gestantes. De acordo com Fernandes et al. (2004), as diferenças podem ser reflexo da interferência da gestação sobre o posicionamento cardíaco, uma vez que o útero aumentado pode deslocar o coração (Barcellos, 2005).

O intervalo QT compreende o período da despolarização ventricular até sua completa repolarização, sendo inversamente proporcional à frequência cardíaca (Diniz et al., 2011). No presente estudo, verificou-se menor valor de duração do intervalo QT no G10 $(\mathrm{P}=0,018)$, possivelmente por causa do maior valor de frequência cardíaca.

Concluiu-se que os parâmetros eletrocardiográficos podem se modificar ao longo da gestação por influência de alterações hemodinâmicas, bem como pela alteração do posicionamento cardíaco nas éguas Crioulas. Ainda, a gestação dessas éguas não causou arritmias importantes ou distúrbios de condução elétrica, sendo predominantes o ritmo sinusal normal e a taquicardia sinusal.

Palavras-chave: cardiologia, eletrocardiograma, equinos, gestação 


\begin{abstract}
Pregnancy can cause several physiologic changes in mares, including modifications in cardiac output and heart positioning. Therefore, these changes could influence electrocardiographic parameters in various stages of pregnancy. This study aimed to evaluate the electrocardiogram of Crioulo mares in different pregnancy stages. Nine non pregnant mares (GO), 11 mares at three months (G3), 11 mares at six months (G6) and 11 mares at 10 months of pregnancy (G10) were submitted to electrocardiographic evaluation. There was higher $(P=0,002)$ heart rate of $G 10(60.45 \pm 12.16 \mathrm{bpm})$ when compared to other groups $(G 0=45.67 \pm 10.75 \mathrm{bpm} ; G 3=44.91 \pm 7.91 \mathrm{bpm} ; G 6=46.91 \pm 9.12 \mathrm{bpm})$, probably because of an increase in cardiac output to uterine perfusion at the end of pregnancy. Normal sinus rhythm was predominant in most of mares of groups G0, G3 and G6, and sinus tachycardia in most of G10 mares. In conclusion, changes in hemodynamics and in cardiac position can influence on the electrocardiogram of pregnant Crioulo mares, but do not cause relevant rhythm or conduction disturbs.
\end{abstract}

Keywords: cardiology, electrocardiogram, equine, pregnancy

\section{AGRADECIMENTOS}

Os autores gostariam de agradecer ao Sr. Marlus Arruda e aos funcionários da Cabanha Morro Chato, por permitirem a realização do estudo, e à FAPESC pelo apoio financeiro.

\section{REFERÊNCIAS}

BARCELLOS, G.A. Achados eletrocardiográficos na gravidez normal. Revista da Sociedade de Cardiologia do Rio Grande do Sul, ano XIV, v.14, p.1-3, 2005.

DINIZ, M.P.; MICHIMA, L.E.S.; FERNANDES W.R. Estudo eletrocardiográfico de equinos de salto sadios. Pesq. Vet. Bras., v.31, p.355-361, 2011.

FERNANDES, W.R.; LARSSON, M.H.M.A; ALVES, A.L.G. et al. Características eletrocardiográficas em equinos clinicamente normais da raça Puro Sangue Inglês. Arq. Bras. Med. Vet. Zootec., v.56, p.143-149, 2004.

FREGIN G.F. Medical evaluation of the cardiovascular system. Vet. Clin. North Am. Equine Pract., v.8, p.329-346, 1992.

História do cavalo Crioulo. ABCCC (Associação Brasileira de Criadores de Cavalo Crioulo). Disponível em: http://www.cavalocrioulo.org.br/ studbook/historia. Acessado em: 8 jun. 2017.

PASCON, J.P.E.; SANTOS, F.P.; PEREIRA, D.T.P. et al. Estudo eletrocardiográfico de éguas da raça Crioula. Pesq. Vet. Bras., v.35, p.319323, 2015.
PATTESON, M. Equine cardiology (electronic version), 2002. Disponível em: http://www.provet.co.uk/equinecardiology/. Acessado em: 07 jun. 2017.

PICON, J.D.; SÁ, A.N.P.O.A. Alterações hemodinâmicas da gravidez. Revista da Sociedade de Cardiologia do Rio Grande do Sul, ano XIV, v.5, p.1-2, 2005.

SCHADE, J.; SCHADE, M.F.S.; FONTEQUE, J.H. Auscultatory and electrocardiographic characteristic of Crioulo horses. Pesq. Vet. Bras., v.34, p.281-289, 2014.

SELLNOW, L. Fetal development and foal growth. The Horse, 1999. Disponível em: http://www.thehorse.com/articles/10257/fetaldevelopment-and-foal-growth. Acessado em: 07 jun 2017.

WINTER, G.H.Z.; RUBIN, M.I.B.; DE LA CORTE, F.D. et al. Gestational length and first postpartum ovulation of Criollo mares on a stud farm in Southern Brazil. J. Equine Vet. Sci., v.27, p.531-534, 2007.

YONEZAWA, L.A.; BARBOSA, T.S.; KOHAYAGAWA, A. Eletrocardiograma do equino. Rev. Ciênc. Agrovet., v.13, p.84-93, 2014. 